\title{
SOME FORMULATION ISSUES IN CONSTRUCTING METAMODELS
}

\author{
Dr. Russell R. Barton \\ Durham University and The Pennsylvania State University \\ 413 Business Building, University Park PA 16802 \\ rbarton@psu.edu
}

\begin{abstract}
Metamodels approximate the mapping of simulation input parameters to simulation outputs, providing a fast-running approximation, and (sometimes) insight on the nature of the response. Sometimes the obvious input variables and model structure give inferior metamodel fidelity. This paper illustrates three cases where the choice has significant impact on the fidelity and usefulness of the metamodel.
\end{abstract}

Keywords: Formulation, Model Parameters, Metamodels

\section{INTRODUCTION}

The simulation community has used metamodels to study the behaviour of computer simulations for more than fifty years (Burdick and Naylor, 1966) although the term was not employed until ten years later. The term for such 'models of a model' was used first by Blanning (1975), then by Kleijnen (1975), who popularized the term and made many significant contributions. Other disciplines refer to such models as response surface or surrogate models. For recent reviews, see Barton (2015) and Klejnen (2017).

There are numerous works devoted to model form and experiment design technique - again see the references above. But on some occasions, the usual approaches fail. In the sections below, three such cases are presented. Section 2 presents a scenario involving prediction of job completion time quantiles with a response function having nonhomogeneous variance. While the form of the Box-Cox variance stabilizing transformation is clear, employing the transformation increases nonlinearity and consequently model complexity. Section 3 focuses on metamodels for network simulations, where routing probabilities are the independent variable in the metamodel. Because each routing probability vector must sum to one, the design space is not full-dimensional. But the design space of the natural reformulation (leave one element of the vector out) has poor spatial structure as the number of components increases. Two alternative formulations have better structure. Section 4 presents more detail on an issue raised in Barton (2005). When seeking a collection of metamodels that are invertible, the choice of responses affects invertibility. The paper concludes with some remarks on awareness of model formulation issues when constructing simulation metamodels.

\section{WHEN A BOX-COX TRANSFORMATION CAN FAIL}

Pedrielli and Barton (2019) examined metamodels for quantiles of job completion time distributions as a function of jobs awaiting processing at each workstation upon release of a new job. Quantiles can be viewed as a guarantee of completion time. In this setting, the new job completion time increases as a function of the number of jobs waiting, and the independent processing times of jobs implies that the variance of completion time also increases as a function of the number of jobs waiting.

There are a number of transformations that can be used for variance stabilizing purposes and to improve the analysis. The family of power transformations proposed by Box and Cox (1964) are of the form: 


$$
y^{\lambda}=\left\{\begin{array}{cc}
\frac{y^{\lambda}-1}{\lambda} & \lambda \neq 0 \\
\log (y) & \lambda=0
\end{array}\right.
$$

where $\lambda$ typically takes on values of -1 (reciprocal), 0 ( $\log$ ), $1 / 2$ (square root), and 2 (square). Statistical software can estimate the value of $\lambda$ for the Box-Cox transformation by the method of maximum likelihood. More simply, a variance stabilizing transformation can be selected via a plot of $\log$ standard deviation of response vs. log mean, assuming that there are multiple replications for each setting for the independent variables. See Chapter 3 of Montgomery (2017) for a detailed discussion of variance-stabilizing transformations.

Barton (2015) illustrated the advantage of a Box-Cox transformation for a simple queueing simulation with response as average waiting time as a function of mean service time. A $\log$ transformation of waiting time reduced heteroscedasticity of the response and also reduced the nonlinearity of the model, improving the $\mathrm{R}^{2}$ of a fitted quadratic metamodel from $82.7 \%$ to $88.9 \%$.

The response function for the job completion time quantile has a different structure that does not share this synergistic characteristic. The nonlinearity in the response arises primarily from the heteroscedastic variance; the mean completion time is a linear function of the jobs awaiting processing ahead of the new job when processing times are exponential, and approaches linearity for other distributions as the number of queued jobs increases. As a result, the impact of the quantile nonlinearity can be small relative to the linear relationship of the mean.

Figure 1 shows residual plots for quadratic regression for untransformed and transformed responses.

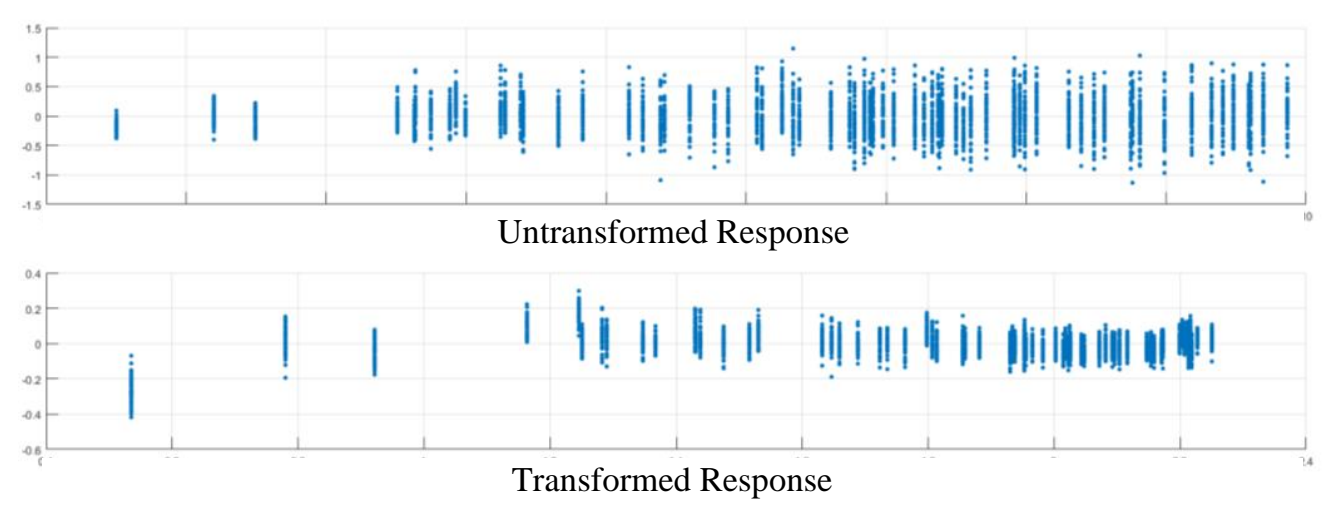

Figure 1 Residuals for Quadratic Metamodel of Completion Time Quantile

The results show the transform-induced nonlinearity at the lower end of the scale for the residuals of the transformed response metamodel - beyond what a quadratic function could capture. While the transformation reduced heteroscedasticity of the response, the predictive ability (over randomly selected points in the design space) of the quadratic metamodel was reduced from that of the metamodel of the untransformed completion time quantiles.

\section{MODELING ROUTING PROBABILITIES}

Barton (2005) developed metamodels for a network design simulation. For these metamodels, routing probabilities were the independent variables. For the general case, a model (and the corresponding metamodel) would have one (outgoing) routing probability vector for each node. A probability vector for a particular node, say $i^{\text {th }}$, might be represented by the vector whose elements were the set $\left\{P_{i j}\right\}$, where $P_{i j}$ is the probability of routing to node $j$ upon leaving node $i$. We will consider the vector for a single node, and so drop the $i$ subscript in what follows. In Barton's network design simulation, there was a single routing node, and probabilities $\left\{P_{j} ; j=1,2,3\right\}$ were parameterized as $\left\{P_{1}, P_{2} /\left(1-P_{1}\right)\right\}$. 
The reduction in cardinality from three to two was necessary to provide a full-dimensional space for the metamodel independent variables, since $P_{1}+P_{2}+P_{3}=1$, implying that the space of values would lie on a two-dimensional hyperplane in 3-space. This is illustrated in Figure 2. This is a characteristic of any routing probability vector, so for metamodeling it is natural to reduce the dimension by one. The simplest and natural strategy is to drop one element of the probability vector from the metamodel, say the last, which results in a full-dimensional design space of Pdim - 1, where Pdim is the cardinality of $\left\{P_{j}\right\}$. The metamodel variables then are $\left\{P_{1}, P_{2}, \ldots, P_{P_{\text {dim }-1}}\right\}$.

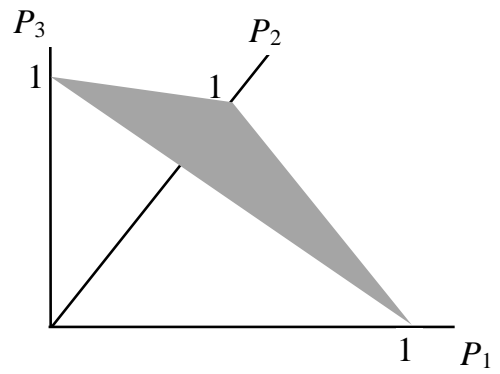

Figure 2 Design Space (shaded) Falls on 2-D Hyperplane for Pdim = 3

When Pdim is small, the parameterization 'All-But-1' works quite satisfactorily for metamodeling. For example, when Pdim $=3$, one has the shaded space shown in Figure 3 for the design region. Note that the shaded region covers one half of the two-dimensional hypercube (e.g., square) $[0,1]^{2}$.

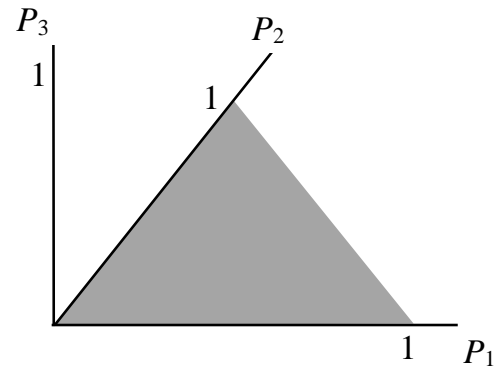

Figure 3 Design Space (shaded) for All-But-1 with Pdim = 3

While the geometry of this structure appears satisfactory, in high dimensions the corresponding shaded region captures an increasingly small (flattened) fraction of the Pdim-1 dimensional hypercube. This results in an ill-conditioned design space, leading to problems in fitting and using the resulting metamodel. This is shown in the computational examples below.

An alternative formulation is possible: embedding the shaded space in Figure 2 (a regular simplex) in Pdim-1 space, and representing each probability vector by its Cartesian coordinates in Pdim-1 space. This is an easy transformation: given the Cartesian coordinates of the simplex vertices, the Cartesian representation of the probability vector is just the convex combination of the coordinates of the simplex vertices, weighted by $\left\{P_{j}\right\}$. Vertices for a regular simplex with edge length $=1$ were defined by Spendley, Hext and Himsworth (1962) for their optimization algorithm. One vertex is the zero vector, each of the other vertices has a representation $(q, q, \ldots, q, p, q, \ldots, q)$ where 


$$
p=\frac{1}{P \operatorname{dim} \sqrt{2}}((P \operatorname{dim}-1)+\sqrt{P \operatorname{dim}}) \text { and } q=\frac{1}{P \operatorname{dim} \sqrt{2}}(\sqrt{P \operatorname{dim}}-1) .
$$

and the position of $p$ varies from 1 to Pdim-1. For Pdim $=3$, the corresponding simplex vertices are shown in Figure 4.

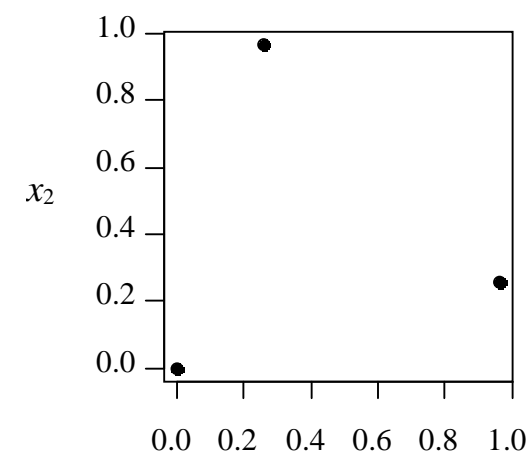

$x_{1}$

Figure 4 Design Space within Vertices of Regular Simplex with Pdim $=3$

An alternative representation permits the dimension of parameter space to remain at Pdim, but relaxes the requirement that the probability vector sum to one. We call this a 'PseudoProbability' vector. The metamodel operates with this vector, while the simulation model uses the normalized version. The result is a response surface that extends the values found on the simplex along rays emanating from the origin. For values close to the origin, spatial variations occur more rapidly. In the computational comparison below, each Pdim-dimensional random vector in the design is multiplied by a (different) sample from $\mathrm{U}(0.5,1.5)$.

\subsection{A Computational Comparison}

Consider a fitting design that is random, with design vectors computed as deviations from the vector with all values $=1 /$ Pdim. Suppose that the deviations for each probability are uniform $+/-.2 /$ Pdim about the 1/Pdim nominal values. In the evaluations below, 1000 design points are generated. It is important to remember that we are generating vectors to use in an experiment design, and the (random) generation process is not the same as generating 1000 'empirical' probability vectors, each based on 1000 samples from the multinomial (, Pdim, (1/Pdim, ..., 1/Pdim) distribution. To illustrate the contrast, for small $n$, empirical values more distant than $+/-.2 /$ Pdim from $1 /$ Pdim are likely for some components of the probability vector. For large $n$, values as far away as $+/-\varepsilon P d i m$ are increasingly unlikely for any fixed $\varepsilon$.

To compare the quality of the different parameterizations, we use a common measure of design quality, the covariance matrix of the design points (e.g. $(X$-Xbar)'(X-Xbar)), where $X$ is the numSamples $\mathrm{x}$ Pdim or Pdim-1 matrix of probability vectors used to fit the metamodel and Xbar is a vector of average values elementwise for the probability vectors in the design. This is equivalent to $X^{\prime} X$ for a design matrix with each probability element centred about its mean across the design.

Figure 5 shows the eigenvalues of the covariance matrices for each parameterization for $P \operatorname{dim}=3$ (on the left) and Pdim $=30$ (on the right). The relative magnitudes are important, and indicate the balance of the design across the spatial dimensions of the probability vector. For a good design all would be approximately equal. The figure shows that using the full probability vector results in a zero eigenvalue, both for Pdim $=3$ and 30. This indicates that the design falls in a Pdim-1 dimensional subspace, problematic for metamodels incorporating the full probability vector. For the Pdim $=3$ case, all three alternative representations perform reasonably well. For Pdim $=30$, only the Cartesian 
representation is satisfactory: both the All-But-1 and the PseudoProbability representations each produce one eigenvalue that is near zero.
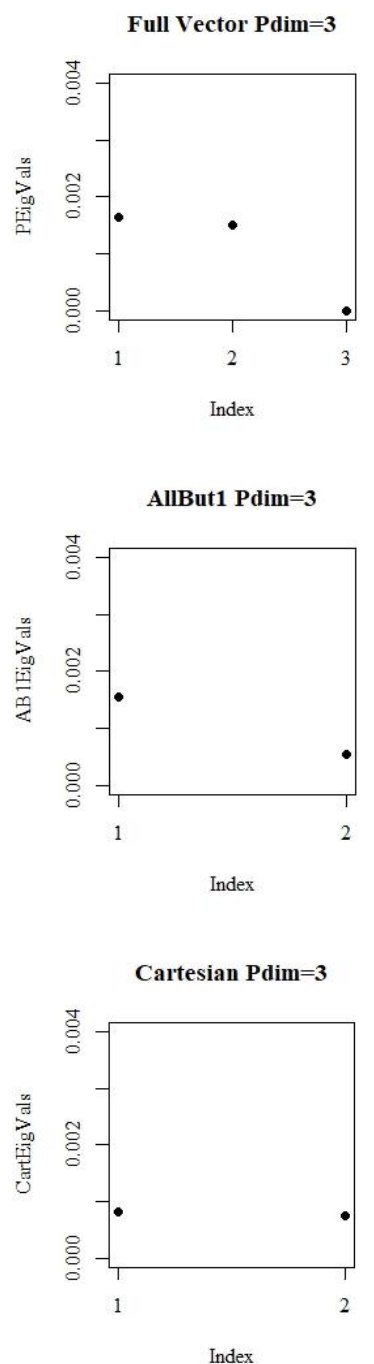

PseudoProb Pdim=3

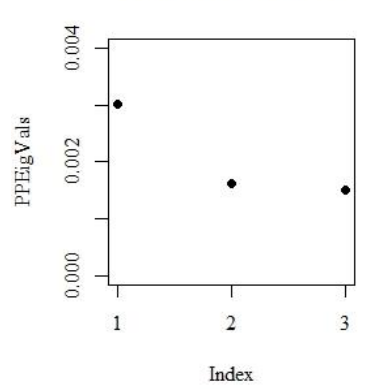

Full Vector Pdim $=30$

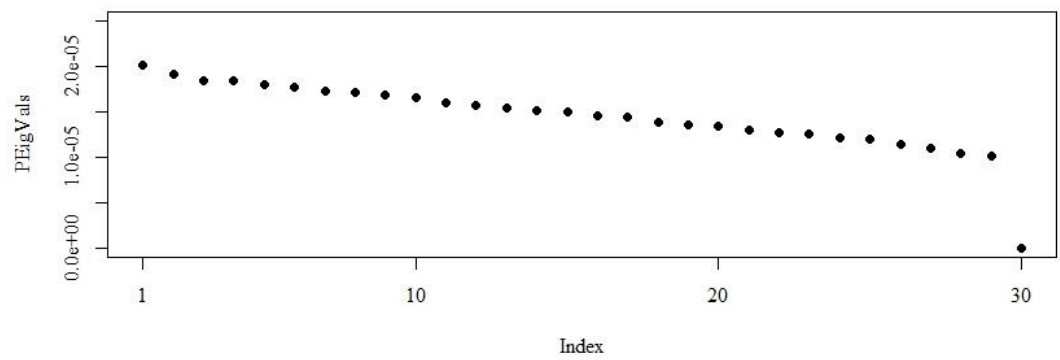

AllBut1 Pdim=30

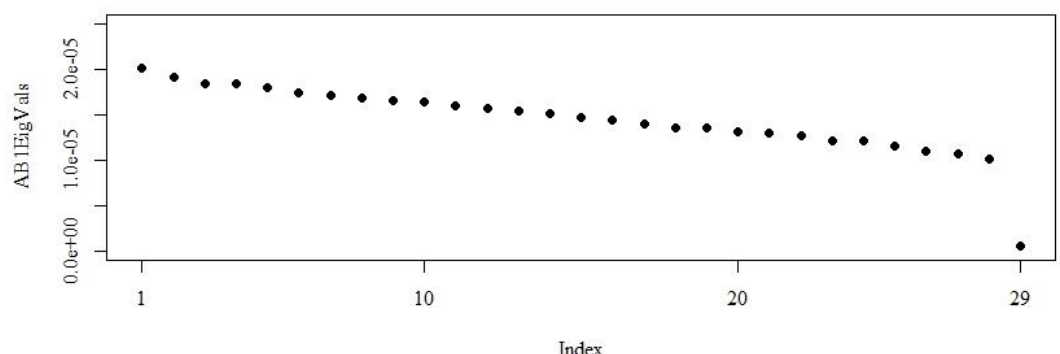

Cartesian Pdim $=\mathbf{3 0}$

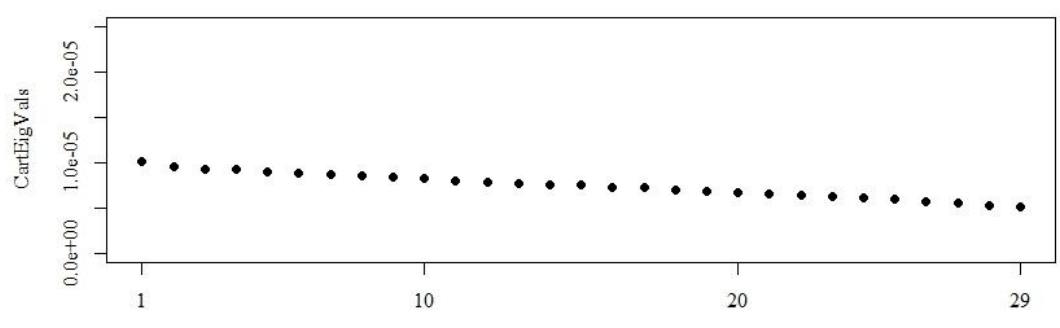

Index

PseudoProb $\mathbf{P d i m}=\mathbf{3 0}$

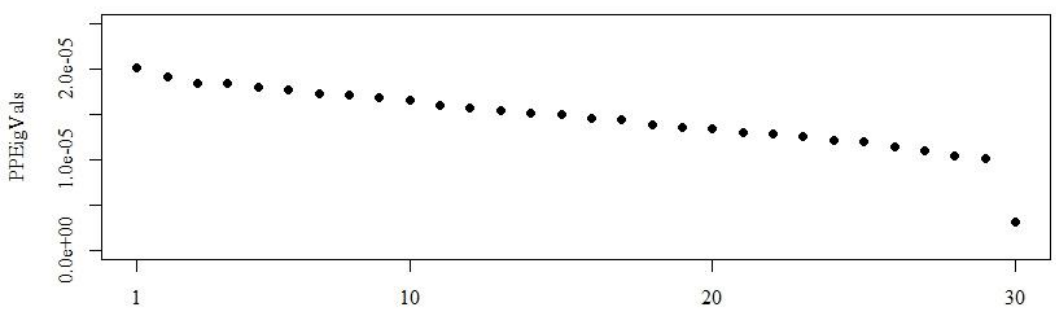

Index

Figure 5 Eigenvalue Plots for Each Probability Parameterization, Pdim = 3, 30

Simulations of routing networks in manufacturing settings may only have a few possible routes from each machining node. But simulations of communications networks and large service operations may have tens or hundreds of routing possibilities at a particular node or point of service. As a consequence, it is important to use the better representation, i.e., the Cartesian representation, when metamodeling with large probability vectors. 


\section{MODELING FOR INVERTIBILITY}

In customer-driven design of systems or products, one has performance targets in mind and would like to determine values for product or system parameters that meet such targets. Simulation models predict performance given design parameter values; meeting a target is done iteratively through an optimization search procedure, typically by optimizing a regression, neural network or other type of approximation metamodel of the computer model. Barton $(2005,2006)$ describes situations that allow inversion of the mapping. Consider a set of $\{\mathrm{X}, \mathrm{Y}\}$ data, where $\mathrm{X}$ and $\mathrm{Y}$ are each $n \times k$ matrices, with a row of $\mathrm{X}$ corresponding to the $k$ design parameter settings for a particular run, and the same row of $\mathrm{Y}$ corresponding to $k$ output measures from one or more simulation runs. While a metamodel can be constructed for each column of $\mathrm{Y}$ as a function of $\mathrm{X}$, it may also be possible to construct a metamodel for each column of $\mathrm{X}$ as a function of $\mathrm{Y}$.

But the selection of the columns of $\mathrm{Y}$, the coordinate functions of the mapping, can affect invertibility. Each coordinate function should be monotonic in the independent $(x)$ variables. This means, for example, decomposing a total cost function into elements that vary monotonically with the elements of $x$. Total cost functions typically have a minimum which is sought, but often they are a sum of monotonic elements. Once can view this as how to parameterize the independent variables for the metamodels from $y$-space to $x$-space. The natural cost representation for the network example in Barton (2005) resulted in a mapping that was not invertible, and an alternative was proposed that led to an invertible mapping. Here we construct a simpler example (with analytic rather than simulation maps) to illustrate the issue.

Imagine making an investment decision on two projects, say 1 , and 2 , affecting different parts of a manufacturing operation. The level of investment for each project can range from 20 units to 65 units independently. Increasing investment reduces operations cost, expressed as a monthly quantity, but increasing investment brings on added debt service costs. Diminishing returns in delay cost savings are observed for both projects, and debt costs increase linearly in cost to first order, but with a secondorder term increasing in the total debt. Figure 6 shows a grid of points in $x$-space, covering the range of investment choices (left side), and the corresponding images in $y$-space, when $y_{1}=$ monthly total cost for project 1 and $y_{2}=$ monthly total cost for project 2 (right side).
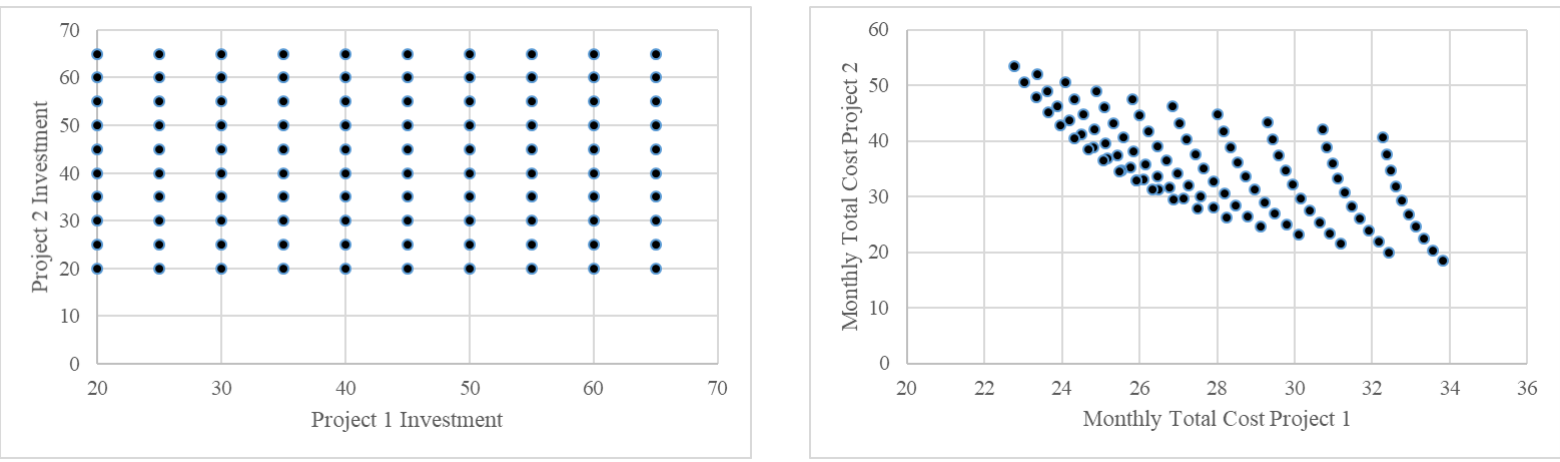

Figure 6 Domain of Investment Decision Space and Corresponding Image with Project Costs

The lower left corner of the cost plot dots in fact overlap. The mapping is not invertible in this area. This is because the total cost function for either project is not invertible. The cost decreases with increasing investment until the diminishing returns are overcome by increasing debt service costs. Instead, one can construct the coordinate functions to be total operations cost for projects 1 and 2, and total debt service costs for both projects. Figure 7 again shows the grid of points in $x$-space, covering the range of investment choices, but the corresponding images in $y$-space, are based on $y_{1}=$ monthly total operations cost for projects 1 and 2 and $y_{2}=$ monthly total debt service cost for projects 1 and 2 .

Because both coordinate functions are monotonic, the mapping is invertible. Note that it is easy to determine total monthly costs from the contour lines on the figure. In this simple two-dimensional 
example, the inverse map can be determined visually. In more complex settings, a fitted inverse metamodel would allow one to choose an investment decision to match any feasible cost profile.
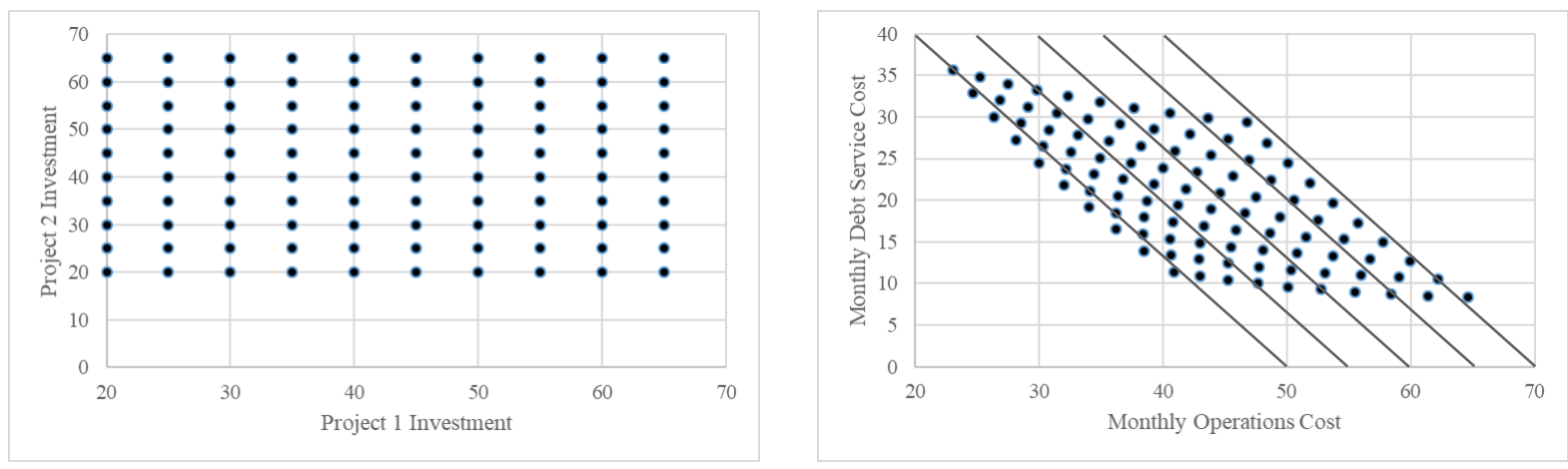

Figure 7 Investment Decision Space and Corresponding Image, using Operations and Debt Costs

\section{SUMMARY}

Researchers and practitioners interested in metamodeling typically focus on the choice of model type and on the experiment design for fitting the metamodel. In some cases, the success of the effort depends on how one chooses to represent the independent variables and dependent variables. First, when facing heterogeneous variance, which often simplifies the response as well. The example here shows that sometimes the response is already a simple function, and the VST can destroy that property. Second, metamodeling with routing probabilities is particularly prone to misparameterization; for more than a few dimensions, probability vectors should be cast in terms of their Cartesian coordinates on the probability simplex. Finally, when one is building a set of metamodels for invertible maps, a good choice of function decomposition can provide invertibility.

\section{ACKNOWLEDGMENTS}

I am grateful to my collaborators on metamodeling projects that led me to these findings. The parameterization issues in fitting metamodels of job completion quantiles arose during research with Giulia Pedrielli at Arizona State University. The parameterization issues in fitting metamodels using routing probabilities arose during research with Eunhye Song at The Pennsylvania State University and Henry Lam at Columbia University. Finally, I thank the reviewers for helpful suggestions to improve the clarity and quality of this paper.

\section{REFERENCES}

Barton, R R (2005). Issues in development of simultaneous forward-inverse metamodels. In Proceedings of the 2005 Winter Simulation Conference, ed. M E Kuhl, N M Steiger, F B Armstrong, and J A Joines, 209-217. Piscataway, N.J.: Institute of Electronic and Electrical Engineers.

Barton, R R (2006). A two-phase maxi-min algorithm for forward-inverse experiment design. In Proceedings of the 2006 Winter Simulation Conference, ed. L F Perrone, F P Wieland, J Liu, B G Lawson, D M Nicol, and R M Fujimoto, 376-381. Piscataway, N.J.: Institute of Electronic and Electrical Engineers.

Barton, R R (2015). Introductory tutorial: simulation metamodeling. in Proceedings of the 2015 Winter Simulation Conference, ed. L Yilmaz, W K V Chan, I Moon, T M K Roeder, C Macal, and M D Rossetti, 1765-1779. Piscataway, N.J.: Institute of Electronic and Electrical Engineers.

Blanning, R W (1975). The construction and implementation of metamodels. Simulation 24: 177-184.

Burdick, D S and Naylor, T H (1966). design of computer simulation experiments for industrial systems. Communications of the ACM 9: 329-339. 
Jacoby, J E and Harrison, S (1962). Multi-variable experimentation and simulation models. Naval Research Logistics Quarterly 9: 121-136.

Kleijnen, J P C (1975). A comment on Blanning's metamodel for sensitivity analysis: the regression metamodel in simulation. Interfaces, 5: 21-23.

Kleijnen, J P C (2017). Regression and kriging metamodels with their experimental designs in simulation: a review. European Journal of Operational Research 256: 1-16.

Montgomery, D C (2017). Design and Analysis of Experiments. $9^{\text {th }}$ ed., John Wiley \& Sons, Hoboken, NJ.

Pedrielli, G and Barton, R R (2019). Metamodel-based quantile estimation for hedging control of manufacturing systems. In Proceedings of the 2019 Winter Simulation Conference, ed. N Mustafee, K-HG Bae, S Lazarova-Molnar, M Rabe, C Szabo, P Haas, and Y-J Son. Piscataway, N.J.: Institute of Electronic and Electrical Engineers.

Spendley, W, Hext, G R, and Himsworth, F R (1962). Sequential application of simplex designs in optimisation and evolutionary operation. Technometrics 4: 441-461.

\section{AUTHOR BIOGRAPHY}

RUSSELL R. BARTON is Distinguished Professor of Supply Chain and Information systems and Professor of Industrial Engineering at the Pennsylvania State University. He is currently a Visiting Professor at Durham University Business School. He received a BS (Hons) in Electrical Engineering from Princeton University and MS and $\mathrm{PhD}$ in Operations Research from Cornell University. He is a fellow of IISE and a Certified Analytics Professional. His research interests include applications of statistical and simulation methods to system design and to product design, manufacturing and delivery. His email address is rbarton@psu.edu. 